Original Paper http://ajol.info/index.php/ijbcs http://indexmedicus.afro.who.int

\title{
Diversité et importance socio-économique des Loranthaceae parasites des plantes ligneuses des Monts Mandara dans la Région de l'Extrême-Nord, Cameroun
}

\author{
Djibrilla MANA $^{1 *}$, Souare KONSALA ${ }^{1}$ et Ibrahima ADAMOU ${ }^{2}$ \\ ${ }^{1}$ Département des Sciences Biologiques, Faculté des Sciences, Université de Maroua, BP: 814 Maroua, \\ Cameroun. \\ ${ }^{2}$ Département des Sciences Biologiques, Faculté des Sciences, Université de Ngaoundéré, BP: 454 \\ Ngaoundéré, Cameroun. \\ *Auteur correspondant ; E-mail: djibrillamana96@gmail.com; Tel. : (+237) 693689363
}

\author{
Received: 29-01-2021 $\quad$ Accepted: 26-04-2021 $\quad$ Published: 30-04-2021
}

\section{RESUME}

Les Loranthaceae constituent des ressources précieuses pour les populations locales africaines. Cependant, peu d'ethnies connaissent l'importance socio-économique des Loranthaceae de par le manque d'études ethnobotaniques sur ces espèces. Afin de contribuer à la valorisation et à la gestion durable des Loranthaceae parasites des plantes ligneuses, une approche associant deux méthodes a été adoptée. L'une, basée sur des relevés de surface et l'autre sur une enquête ethnobotanique relative aux connaissances locales des Loranthaceae dans 9 Arrondissements de la Région de l'Extrême-Nord du Cameroun. Au total, 4 genres (Agelanthus, Globimetula, Phragmanthera et Tapinanthus) et 7 espèces parasites (Agelanthus dodoneifolius, Globimetula braunii, Phragmanthera capitata, Tapinanthus bangwensis, T. belvisii, T. globiferus et T. ophiodes) ont été inventoriés et identifiés dans les Monts Mandara. Il ressort aussi que ces espèces parasites sont utilisées en pharmacopée dans des recettes médicinales et magico-religieuses $(45,56 \%)$, pour traiter des infertilités $(32,70 \%)$ et des troubles mentaux (32,36\%). Les résultats ont aussi montré que Mangifera indica $(35,74 \%)$ et Senna singueana $(34,41 \%)$ sont les hôtes des parasites les plus sollicités. Aussi ont-ils révélé que la commercialisation des Loranthaceae constitue une source de revenu économique et une ressource importante en pharmacopée pour les populations locales des Monts Mandara. Ces résultats pourraient servir de référence pour la recherche de stratégies d'exploitation et de conservation durable de cette diversité végétale.

(C) 2021 International Formulae Group. All rights reserved.

Mots clés : Loranthaceae, Espèces parasites, pharmacopée, Monts Mandara, Cameroun.

\section{Diversity and socio-economic importance of Loranthaceae parasites of woody plants of Mandara Mountains in the Far-North Region, Cameroon}

\section{ABSTRACT}

Loranthaceae are valuable resources for local African populations. However, few ethnic groups know the socio-economic importance of Loranthaceae due to the lack of ethnobotanical studies on these species. In order to contribute to the enhancement and sustainable management of Loranthaceae parasites of woody plants, an approach combining two methods was adopted. One, based on surface surveys and the other on an 
ethnobotanical survey relating to local knowledge of Loranthaceae in 9 Subdivisions of the Far North Region of Cameroon. In total, 4 genera (Agelanthus, Globimetula, Phragmanthera and Tapinanthus) and 7 parasitic species (Agelanthus dodoneifolius, Globimetula braunii, Phragmanthera capitata, Tapinanthus bangwensis, T. belvisii, T. globiferus and T. ophiodes) have been inventoried on the Mandara Mountains. It also appears that these parasitic species are used in pharmacopoeia in medicinal and magico-religious recipes (45.56\%), to treat infertility (32.70\%) and mental disorders (32.36\%). The results also showed that Mangifera indica $(35.74 \%)$ and Senna singueana $(34.41 \%)$ are the hosts of most solicited parasites. They also revealed that the commercialisation of Loranthaceae constitutes a source of economic income and an important resource in pharmacopoeia for the local populations of the Mandara Mountains. These results could serve as a reference of strategies for the sustainable exploitation and conservation of this plant diversity.

(C) 2021 International Formulae Group. All rights reserved.

Keywords: Loranthaceae, parasitic species, pharmacopoeia, Mandara mountains, Cameroon.

\section{INTRODUCTION}

Les Loranthaceae sont des plantes vasculaires épiphytes hémiparasites et chlorophylliennes qui vivent aux dépens d'autres plantes qu'elles parasitent. Ces hémiparasites, une fois fixés sur les branches ou le tronc de l'hôte, grâce à un système endophyte complexe appelé haustorium ou suçoir puisent dans les tissus du sujet parasité, l'eau et les sels minéraux nécessaires pour leur propre développement (Boussim, 2002 ; Dibong et al., 2009). Par ce mode d'infestation, ces Loranthaceae causent de nombreux dommages sur les arbres et les arbustes cultivés ou non, allant de l'envahissement total au dessèchement des branches infestées (Koffi et al., 2014). La taxonomie des Loranthaceae révèle l'existence d'environ 950 espèces, réparties dans 77 genres dans le monde (Polhill \& Wiens, 1998). En Afrique, ces mêmes auteurs dénombrent environ 500 espèces, réparties dans 21 genres. Au Cameroun, les Loranthaceae sont représentées par plus de 26 espèces, réparties dans 7 genres, qui sont: Agelanthus, Englerina, Globimetula, Helixanthera, Phragmanthera, Tapinanthus et Viscum (Dibong et al., 2009). Elles sont présentes dans la plupart des groupements végétaux du Cameroun, y compris les mangroves. Elles sont perceptibles sur les cultures pérennes, les espèces fruitières dans les plantations, des jardins de cases et autres vergers, avec des taux et d'intensités d'infestation souvent très élevés (Dibong et al., 2010 ; Mony et al., 2014). Les espèces ligneuses d'importance environnementale et socio-économique telles que Terminalia sp., Dacryodes edulis, Psidium guajava, Citrus sp. et Vitellaria paradoxa sont attaquées à des degrés divers par les Loranthaceae (Amon et al., 2015 ; Ahamide et al., 2017).

Bien qu'elles soient des plantes parasites, nuisibles des arbres et arbustes cultivés ou non, les Loranthaceae jouent également un rôle socio-économique important pour de nombreuses populations rurales en Afrique subsaharienne (Dibong et al., 2009). En effet, elles sont utilisées dans des préparations médicamenteuses pour traiter diverses affections tels que le cancer, l'hypertension, l'hypotension, le diabète, les hépatites, les accidents vasculo-cérébraux, la stérilité, les maladies microbiennes et les troubles mentaux (Kouyaté, 2005 ; Ahamide et al., 2015, Amon et al., 2017). Elles sont également utilisées à des fins mystiques par les tradipraticiens. $\mathrm{Au}$ Cameroun, les plantes médicinales en général et les Loranthaceae en particulier, sont vendues régulièrement sur les marchés des centres urbains et ruraux en différentes saisons de l'année. Elles constituent des ressources précieuses pour de nombreuses populations des zones rurales en Afrique dont $80 \%$ de ces populations utilisent encore les plantes à caractère médicinal pour leur soin de santé primaire (Dibong et al., 2010 ; Adomou et al., 2012). Aujourd'hui, au-delà de leurs utilisations dans le traitement de différentes affections, l'exploitation durable des Loranthaceae est d'un grand intérêt pour les communautés locales à cause de ses retombées économiques par la commercialisation des 
bottes des espèces récoltées. Malgré l'importance des Loranthaceae en pharmacopée, à l'état actuel des connaissances, très peu d'études ont été réalisées sur les Loranthaceae et l'influence des collines sur leur distribution dans la Région de l'ExtrêmeNord, Cameroun (Souare et al., 2020). Cette étude vise donc à évaluer la diversité spécifique des Loranthaceae parasites des plantes ligneuses des Monts Mandara dans la Région de l'Extrême-Nord du Cameroun, déterminer l'influence des collines sur leur distribution et relever leur importance socio-économique.

\section{MATERIEL ET METHODES Milieu d'étude}

L'étude a été conduite sur les collines des Monts Mandara situés dans la zone soudano-sahélienne du Cameroun, de coordonnées géographiques $10^{\circ} 00^{\prime} 00^{\prime}$ " et $11^{\circ} 00^{\prime} 00^{\prime \prime}$ de latitude Nord et $13^{\circ} 30^{\prime} 00^{\prime \prime}$ et $14^{\circ} 30^{\prime} 00^{\prime}$ " de longitude Est (Figure 1). D'une superficie de $7660 \mathrm{~km}^{2}$, les Monts Mandara couvrent les Départements du Mayo-Sava et Mayo-Tsanaga, l'Arrondissement de Méri dans le Diamaré et l'Arrondissement du MayoOulo dans le Mayo-Louti. Ils forment une vaste plaine à l'Est et au Nord et un ensemble de massifs montagneux appelés Monts Mandara dans sa partie Ouest le long de la frontière nigériane. La zone d'étude est caractérisée par un régime climatique de type soudanosahélien. La pluviométrie est de type monomodal de durée et d'intensité variant en moyenne entre 800 et $1000 \mathrm{~mm} / \mathrm{an}$ (Gerhard, 2003). La température moyenne annuelle est de $28^{\circ} \mathrm{C}$ (Maïnam, 1999). Le sol est du type sabloargileux et sableux. La formation végétale est du type soudano-sahélien caractérisée par la steppe à dominance d'arbustes épineux et par son extrême morcellement dû à des conditions naturelles et à l'action anthropique. La population de la zone est estimée en 2005 à environ 1165700 habitants (BUCREP, 2005), dominée par les ethnies Mafa, Moufou, Hide, Foulbé (peulh), Maba et Woula. Les principales activités économiques menées sont l'agriculture, le commerce, l'élevage et l'artisanat.

\section{Méthodes \\ Collecte de données}

Deux types de données ont été collectés. Il s'agit des données d'inventaire des Loranthaceae et des données d'enquêtes ethnobotaniques.

\section{Inventaire des Loranthaceae}

Pour l'inventaire des Loranthaceae, la méthode de relevés de surface qui consiste à recenser les espèces de Loranthaceae rencontrées dans des sous-parcelles de $50 \mathrm{~m} \mathrm{x}$ $10 \mathrm{~m}$ dans chacune des 18 parcelles de $50 \mathrm{~m} \mathrm{x}$ $30 \mathrm{~m}$ a été adoptée. Ces parcelles sont disposées du bas au sommet des collines et distancées de $100 \mathrm{~m}$ à l'horizontale et de $160 \mathrm{~m}$ à la verticale sur chacun des 2 versants (Est et Ouest). Au total, 540 parcelles (15 collines x 2 versants x 18 parcelles) ont été prospectées. Le dispositif expérimental (Figure 2) est un Splitplot à 2 répétitions. Les collines sont les traitements principaux, les Loranthaceae sont les traitements secondaires et les versants sont les répétitions. L'unité expérimentale est constituée de 18 parcelles.

Pour évaluer la distribution spatiale des Loranthaceae sur les Monts Mandara, 15 collines d'au moins $1000 \mathrm{~m}$ de hauteur ont été sélectionnées à raison d'une colline à chaque $10 \mathrm{~km}$, sur l'ensemble des Monts Mandara de $150 \mathrm{~km}$ de longueur totale et d'orientation Nord-Sud. L'ensemble des 15 collines matérialisé par $\mathrm{C} 1, \mathrm{C} 2, \mathrm{C} 3, \mathrm{C} 4, \mathrm{C} 5, \mathrm{C} 6, \mathrm{C} 7, \mathrm{C} 8$, $\mathrm{C} 9, \mathrm{C} 10, \mathrm{C} 11, \mathrm{C} 12, \mathrm{C} 13, \mathrm{C} 14$ et $\mathrm{C} 15$ constituent nos sites de collecte de données d'inventaire et sont localisées à Mora $(\mathrm{C} 1$ C3), Tokombéré (C4), Soulede-Roua (C5), Meri (C6), Mokolo (C7 - C9), Mogodé (C10), Hina (C11), Bourha (C12 - C13) et Mayo-Oulo (C14 - C15). Sur chacune des 15 collines, les parasites et leur hôte ont été inventoriés sur chacun des 3 transects délimités.

Sur chaque plante hôte, toutes les touffes des parasites ont été localisées, dénombrées et identifiées à l'œil nu ou à l'aide des jumelles pour les parasites localisés au niveau des cimes des grands arbres ou en utilisant le guide botanique (Arbonnier, 2009), comme l'ont fait Koffi et al. (2014). La clé de détermination des espèces de Loranthaceae 
utilisée par Houénon (2012) a été utilisée pour identifier les espèces de Loranthaceae. Pour les espèces (parasites et hôtes) non identifiées sur le terrain, les images sont prises à l'aide d'un appareil photo numérique ou un échantillon d'organe de plante (tiges, feuilles, fleurs ou fruits) est prélevé pour identification à l'herbier ou par les experts.

\section{Enquêtes ethnobotaniques}

Pour bien structurer le questionnaire, une visite de prospection a été réalisée en vue de répertorier les acteurs tels que les tradithérapeutes, les éleveurs, les bergers, les responsables des vergers et les paysans ayant une connaissance des espèces parasites de la famille des Loranthaceae et de leurs utilisations. Cette préenquête a été réalisée avec l'aide des chefs traditionnels. Après quoi, 9 Arrondissements des 4 Départements (Diamaré, Mayo-Sava, Mayo-Tsanaga et Mayo-Louti) que couvrent les Monts Mandara ont été retenus pour réaliser les enquêtes, entre septembre et octobre 2018, pendant le repos des paysans à la fin de la saison des pluies. Les enquêtes ont éte menées auprès de 237 personnes composées de 195 hommes et 42 femmes appartenant à 25 ethnies. La connaissance de bonnes informations sur les Loranthaceae et leurs utilisations en pharmacopée a été le seul critère de choix de ces personnes. L'entretien direct a été adopté pour la collecte des données (Jiofack et al., 2009). Ces enquêtes ont été menées au cours des entretiens semi-structurés avec les personnes choisies sur la base des questions ouvertes, fermées et orientées, en langue locale avec l'aide d'un interprète pour faciliter la communication avec les acteurs. Les principales questions portaient sur la connaissance des Loranthaceae et leurs utilisations en pharmacopée.

\section{Traitement des données}

\section{Calculs de la densité et des indices de diversité}

La densité spécifique des espèces a été calculée selon la formule suivante: $\mathrm{D}=\mathrm{N} / \mathrm{S}$,
Où $\mathrm{N}$ est le nombre d'individus du milieu d'étude et $\mathrm{S}$, la surface occupée par ces individus.

L'indice de Shannon des parasites a été calculé selon la formule suivante:

$\mathrm{H}^{\prime}=-\sum \mathrm{ni} / \mathrm{N} \log _{2} \mathrm{ni} / \mathrm{N}$,

Où $\mathrm{H}^{\prime}$ est l'indice de diversité de Shannon, ni est le nombre d'individus de l'espèce $\mathrm{i}$ et $\mathrm{N}$, le nombre total d'individus de toutes les espèces confondues.

A partir de cet indice, l'Equitabilité de Piélou (E) a été calculée selon la formule suivante: $\mathrm{E}=\mathrm{H}^{\prime} / \log _{2} \mathrm{~N}$,

Où $\mathrm{H}^{\prime}$ est l'indice de diversité de Shannon - Weaver et N, le nombre d'espèces.

Le pourcentage de chaque catégorie d'usage a été calculé selon la formule suivante comme l'ont fait Nabila et al. (2012): C $=\frac{\mathrm{n}}{N} \times 100$,

Où $\mathrm{C}$ est le pourcentage d'une catégorie donnée, $\mathrm{n}$ est le nombre de personnes ayant donné une réponse positive pour une espèce $\mathrm{i}$ et $\mathrm{N}$, l'effectif total de l'échantillon.

La valeur d'usage ethnobotanique unitaire d'une espèce $\mathrm{i}$ pour une catégorie d'usage c (VUU) et la valeur d'usage ethnobotanique totale (VUT) ont été calculées pour les espèces de phorophytes les plus sollicitées suivant la méthode de calcul utilisée par Lougbegnon et al. (2011): VUU $=\frac{\mathrm{U}}{N} \times 100$ ; VUT $=\sum$ VUU,

Où VUU est la valeur d'utilisation (\%) unitaire d'une espèce $i$, $U$ est le nombre d'usages médicinaux où l'espèce hôte est mentionnée, $\mathrm{N}$ est le nombre d'informateurs ayant mentionné l'espèce i. VUT est la valeur d'usage ethnobotanique totale de l'espèce i.

\section{Analyse statistique}

L'analyse de variance (ANOVA) à une voie a été utilisée pour comparer les sites d'étude, suivie par le test de Duncan au seuil de $5 \%$ pour la comparaison des moyennes. La distribution spécifique des espèces sur les collines et leur corrélation ont été réalisées à l'aide de l'analyse en composantes principales des variables. Ces tests ont été réalisés à l'aide du tableur EXCEL 2016 et du logiciel XLSTAT 2007. 


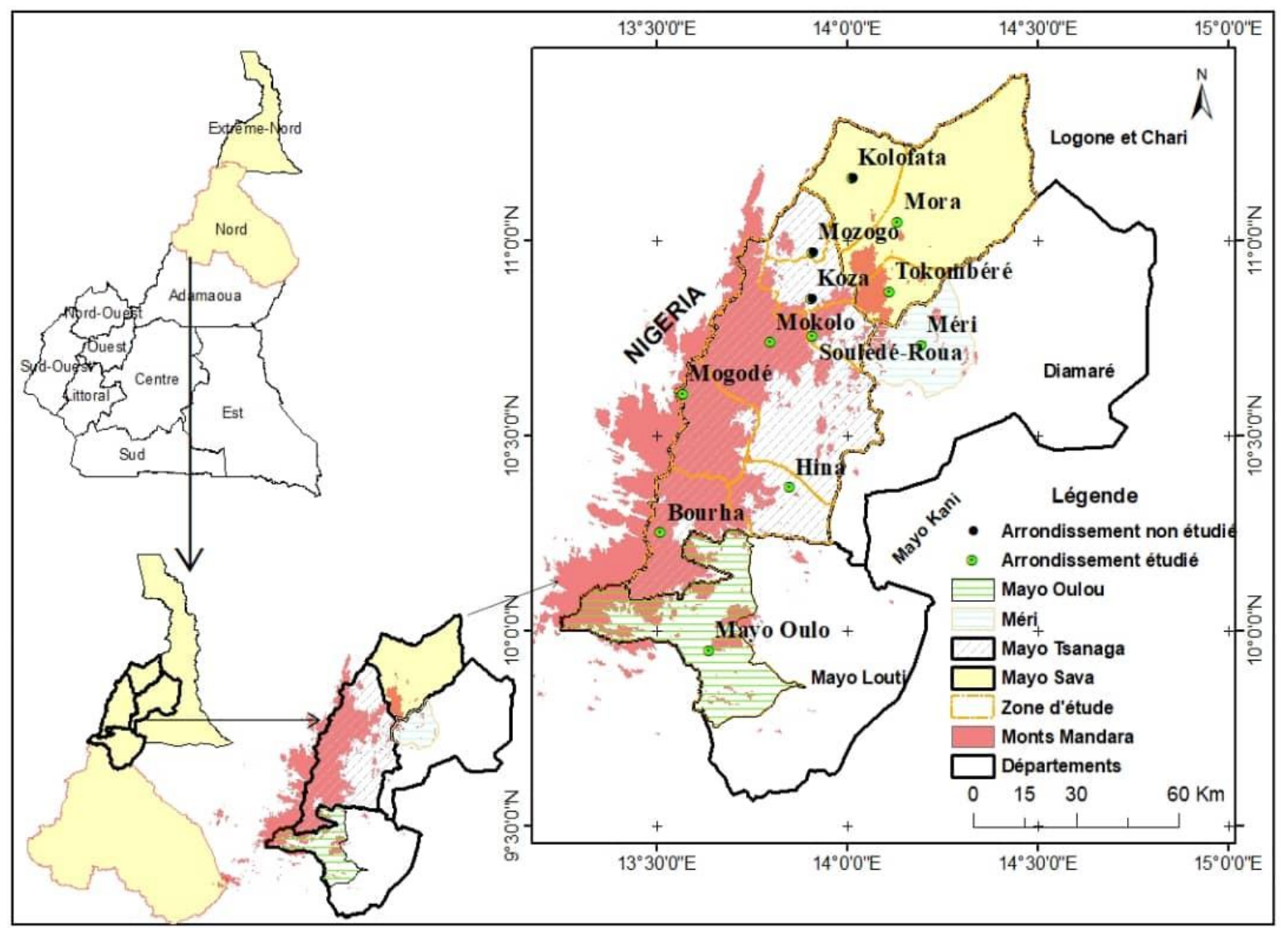

Figure 1: Carte de localisation de la zone d'étude.

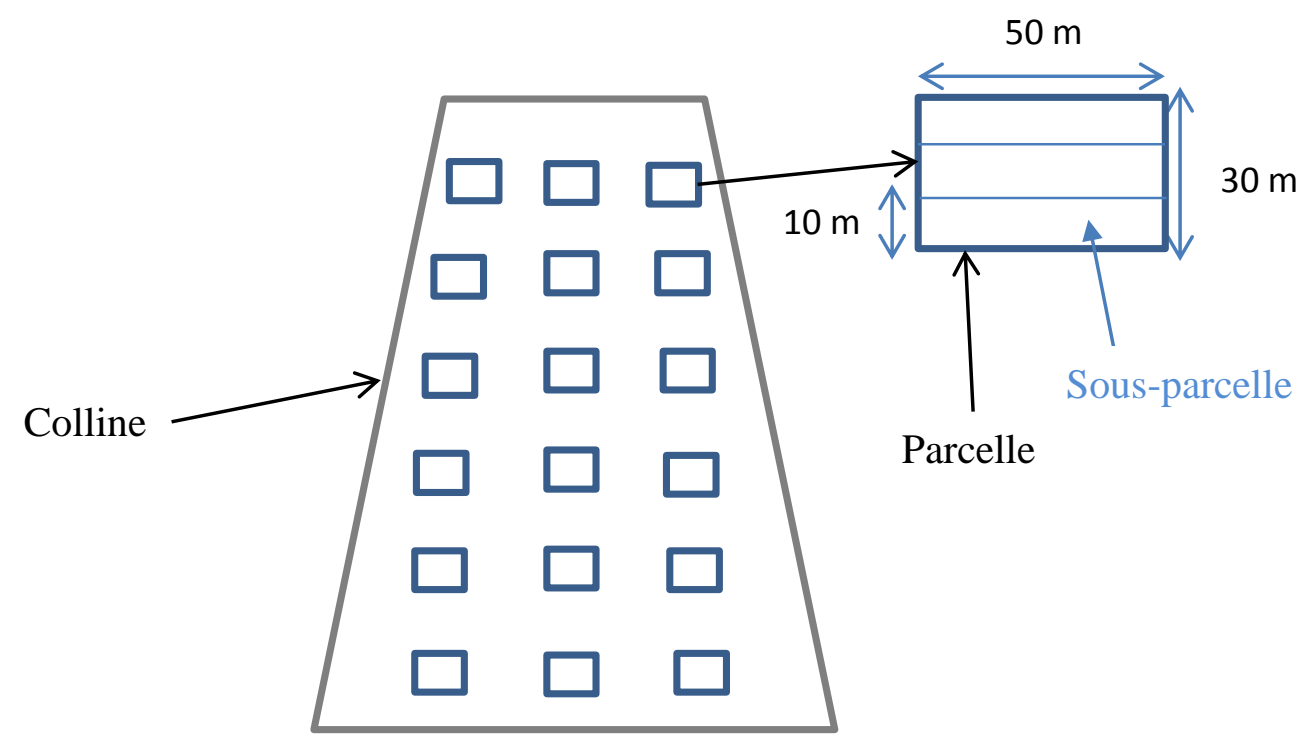

Figure 2: Plan expérimental. 


\section{RESULTATS \\ Composition taxonomique des Loranthaceae}

$\mathrm{Au}$ total, 7 espèces de Loranthaceae parasites des plantes ligneuses ont été recensées sur les 15 collines des Monts Mandara, avec une densité moyenne de 432,38 $\pm 41,22$ ind./ha. Ce sont: Agelanthus dodoneifolius, Globimetula braunii, Phragmanthera capitata, Tapinanthus bangwensis, T. belvisii, T. globiferus et $T$. ophiodes. Elles se répartissent dans 4 genres, à savoir Agelanthus, Globimetula, Phragmanthera et Tapinanthus. Le genre Tapinanthus renferme 4 espèces. La densité et la distribution de ces espèces parasites sont variables (Tableau 1). Parmi ces espèces parasites inventoriées, $T$. globiferus est la plus abondante avec une densité de 609,75 ind./ha. Viennent ensuite A. dodoneifolius et $T$. ophiodes avec des densités moyennes respectives de 549,97 et 439,95 ind./ha. $G$. braunii est l'espèce la moins abondante avec une densité moyenne de 132,18 ind./ha.

\section{Distribution des Loranthaceae sur les collines}

La distribution des espèces sur les collines est variable (Tableau 1). Trois espèces de Loranthaceae (A. dodoneifolius, $T$. globiferus et $T$. ophiodes.) se rencontrent sur toutes les 15 collines étudiées avec une abondance variable en fonction des collines. Parmi ces 3 espèces, A. dodoneifolius et $T$. globiferus sont plus abondantes sur la colline 8 avec des valeurs de densité respectives de l'ordre de 702,22 et 813,33 ind./ha. Par ailleurs, elles sont moins abondantes sur la colline 15 (C15) pour A. dodoneifolius (401,11 ind./ha) et sur la colline 1 (C1) pour $T$. globiferus (298,70 ind./ha). Quant à $T$. globiferus, elle est plus abondante sur la colline 4 (C4) avec une densité de 539,26 ind./ha et faible sur la colline $1(\mathrm{C} 1)$ avec une densité de 298,70 ind./ha. Les 4 autres espèces sont distribuées sur 5 à 9 collines selon l'espèce.
La Figure 4 présente la dispersion des espèces de Loranthaceae sur l'ensemble des 15 collines étudiées. L'axe F1 qui contribue à $81,94 \%$ de la répartition des Loranthaceae sur les collines sépare les espèces les plus abondantes et présentes sur toutes les collines comme T. globiferus, A. dodoneifolius et $T$. ophiodes du côté droit et celles les moins abondantes et présentes sur un nombre faible des collines comme G. braunii du côté gauche. Les trois autres espèces de Loranthaceae ( $T$. belvisii, $T$. bangwensis et $P$. capitata) ont leur répartition et leur abondance moyennes.

Les collines varient entre elles en fonction du nombre d'espèces de Loranthaceae et leur abondance moyenne (Tableau 1). La colline 8 (C8) abrite toutes les 7 espèces parasites, alors que la colline $1(\mathrm{C} 1)$ n'a que 3 espèces rencontrées, à savoir: A. dodoneifolius, $T$. globiferus et $T$. ophiodes. Les autres collines enregistrent entre 4 et 6 espèces de Loranthaceae. Par contre, la colline 1 (C1) qui est la plus faible en termes de nombre de touffes est la plus abondante en Loranthaceae avec une densité de 496,23 ind./ha. Elle est suivie de la colline 2 (C2) et 5 (C5), avec des valeurs de densités respectives de 482,96 et 476,85 ind./ha. La colline 15 (C15) est la plus faible en densité des Loranthaceae (353,11 ind./ha). Elle abrite cinq espèces à savoir: $A$. dodoneifolius, $P$. capitata, T. belvisii, T. globiferus et $T$. ophiodes.

L'indice de diversité de Shannon calculé varie entre les collines (Tableau 2). Il varie de 1,25 bit sur la colline 1 (C1) à 1,90 bit sur la colline 8 (C8). Cet indice est faible pour toutes les collines, inférieur à 2 bits. Quant à l'Equitabilité de Piélou, il varie de 0,632 sur la colline $1(\mathrm{C} 1)$ à 0,763 sur la colline $8(\mathrm{C} 8)$.

La Figure 5 présente les corrélations entre les différentes collines. L'axe F1 qui contribue à $81,94 \%$ explique la distribution des Loranthaceae sur les collines. Cet axe est corrélé avec les collines, en particulier avec les collines $1(\mathrm{C} 1)$ et 5 (C5), qui présentent une grande densité d'individus des Loranthaceae, de l'ordre respectif de 496 et 476 ind./ha. Les 
collines $1(\mathrm{C} 1)$ et $5(\mathrm{C} 5)$, les collines $3(\mathrm{C} 3)$ et 7 (C7), et les collines 6 (C6) et 9 (C9) montrent respectivement une corrélation positive et hautement significative $(\mathrm{P}<0,001)$ avec des coefficients de corrélation (r) respectifs de l'ordre de 0,$996 ; 0,989$ et 0,976 . Par contre, les corrélations entre les collines $12(\mathrm{C} 12)$ et 11 (C11), entre les collines 12 (C12) et 15 (C15) et entre les collines 12 (C12) et 13 (C13) ne sont pas significatives $(\mathrm{P}>0,05)$, avec des coefficients de corrélation de 0,$379 ; 0,387$ et 0,464 respectivement. Le nombre d'espèces recensées sur la colline 12 (6 espèces) est plus grand que celui sur les trois collines 11 (C11), 13 (C13) et 15 (C15), qui est de 5 espèces. La densité des individus de la colline $12(390,74$ ind./ha) est située entre celles des collines 13 (379,41 ind./ha) et 15 (353,11 ind./ha) et celle de la colline 11 (461,93 ind./ha).

\section{Catégories d'usages, valeurs d'usages et importances économiques des Loranthaceae}

Les résultats des enquêtes montrent que les paysans connaissent les espèces de Loranthaceae. Chaque espèce de Loranthaceae est désignée par le nom de l'hôte qui l'héberge. Ils révèlent aussi que les Loranthaceae sont utilisées dans des préparations médicamenteuses pour soigner presque toutes les affections. Elles sont employées également dans des recettes mystiques pour combattre des maux, d'où leur appellation par les personnes interviewées sous le vocable de «plante qui guérit tous les maux ».

Les usages des Loranthaceae en pharmacopée sont nombreux et varient selon les paysans interrogés (Tableau 3). Les enquêtes ont révelé que les espèces de plantes de la famille des Loranthaceae sont plus utilisées au plan magico-religieux que médicinal avec un pourcentage de réponses de $45,56 \%$. Elles sont utilisées dans des recettes médico-magico-religieuses pour traiter les cas d'infertilité masculine et féminine $(32,70 \%)$ et des troubles mentaux $(32,36 \%)$. Il ressort aussi que les maladies chroniques $(26,79 \%)$ et les troubles digestifs $(25,86 \%)$ sont faiblement traités à partir des espèces de Loranthaceae.

La valeur d'usages totale (VUT) en pharmacopée des Loranthaceae varie significativement $(\mathrm{P}<0,001)$ en fonction des plantes hôtes qui les portent, le sexe et le pratiquant (Tableau 4). De manière globale, la valeur d'usages des Loranthaceae varie de 22,32 à $35,70 \%$. Les Loranthaceae se développant sur Mangifera indica $(35,74 \%)$ et Senna singueana $(34,41 \%)$ enregistrent des valeurs d'usages plus élevées et contrairement à celles récoltées sur Kigelia africana. Les Loranthaceae des autres plantes hôtes sont moyennement recherchées pour leurs usages.

Les résultats des enquêtes ont révélé que les Loranthaceae sont une source de revenu pour les tradithérapeutes et les tradipraticiens grâce à la vente des différents organes de ces plantes et leur utilisation dans le traitement de diverses affections (Tableau 5). Il ressort que le revenu journalier des acteurs rencontrés varie entre 200 et 300 FCFA selon la nature des produits issus des Loranthaceae vendus et entre 500 et 1000 FCFA selon la pratique. Les résultats ont montré aussi que les exploitants des Loranthaceae vendent en moyenne 310000 FCFA par mois, soit en moyenne un revenu annuel de 1200000 FCFA. Aussi, ont-ils révélé que les pratiques mystiques assurent plus de revenu aux tradithérapeutes que les ventes simples des produits dérivés des Loranthaceae. En effet, les pratiques magicoreligieuses rapportent annuellement plus de 400000 FCFA que les affections traitées au plan médicinal (350 000 FCFA). On note que les ventes de bottes d'espèces de Loranthaceae rapportent 250000 FCFA de revenu mensuel et autres poudres de ces plantes, 200000 FCFA de revenu par mois. Les résultats révèlent également que les espèces de Loranthaceae transformées en poudre est la forme la moins recherchée par les consommateurs. 

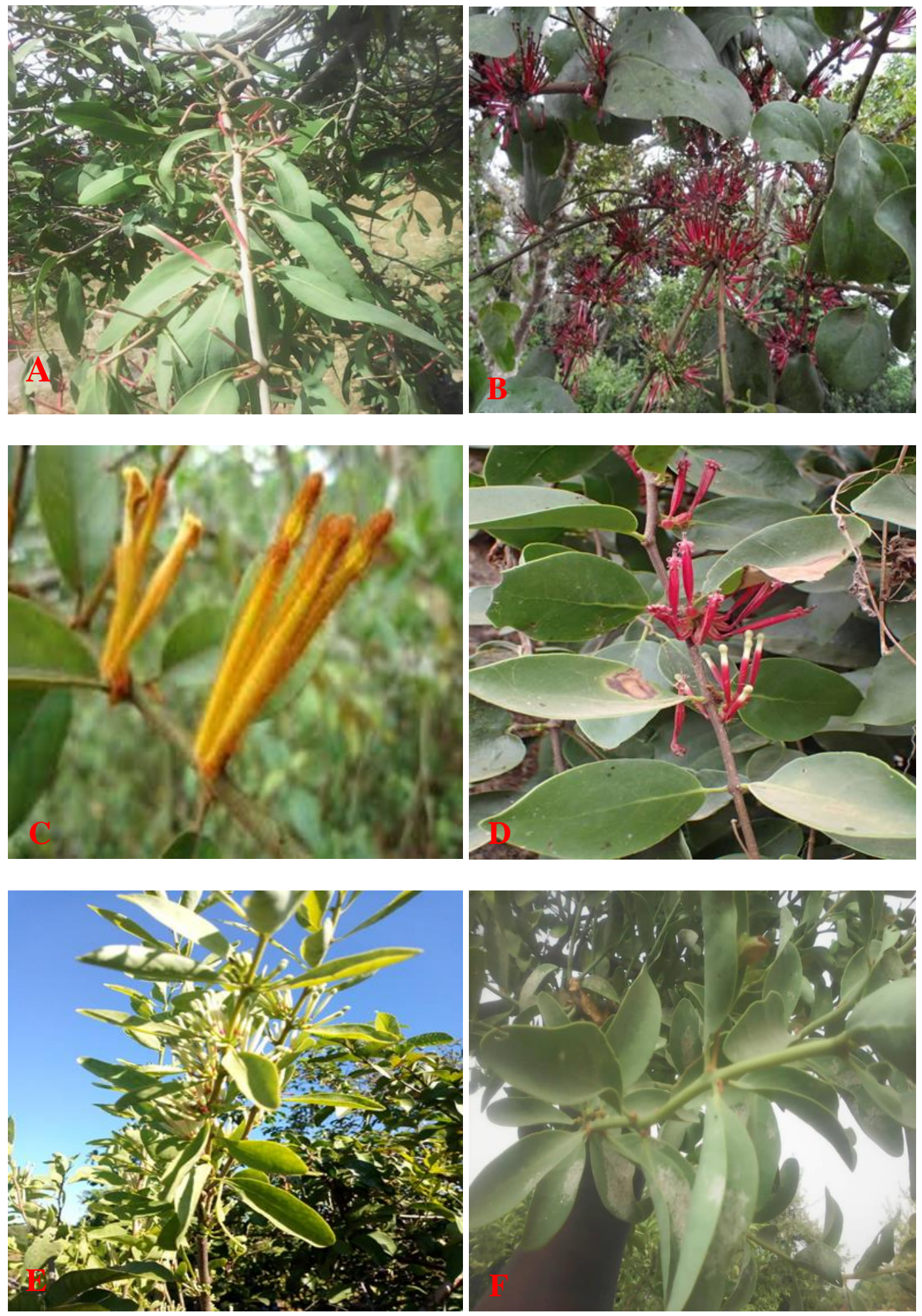


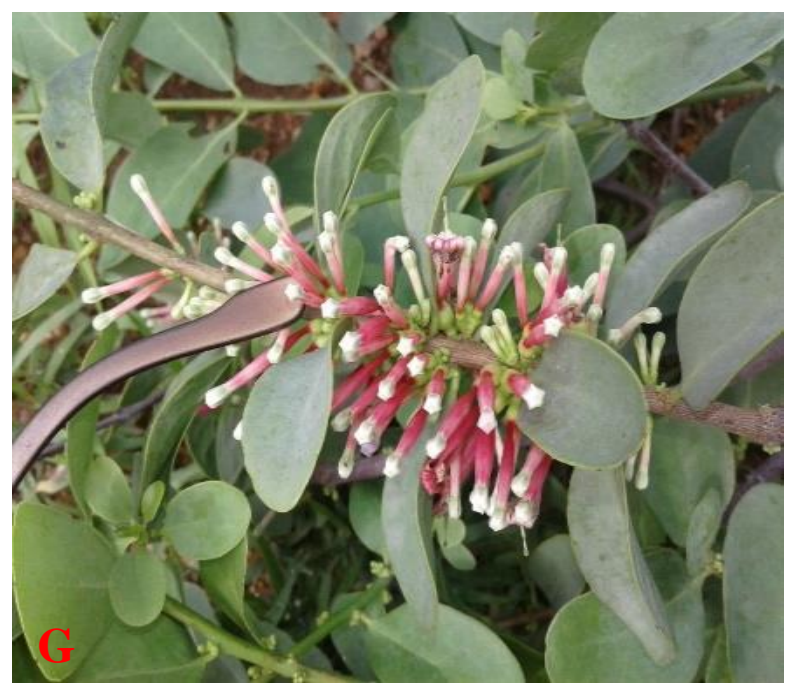

Figure 3: Espèces de Loranthaceae inventoriées sur les Monts Mandara.

Légende : A: Agelanthus dodoneifolius, B: Globimetula braunii, C: Phragmanthera capitata, D: Tapinanthus bangwensis, E: Tapinanthus belvisii, F: Tapinanthus globiferus et G: Tapinanthus ophiodes.

Tableau 1: Composition et densité (ind./ha) spécifiques des Loranthaceae sur les 15 collines.

\begin{tabular}{|c|c|c|c|c|c|c|c|c|}
\hline \multirow{2}{*}{ Collines } & \multirow[b]{2}{*}{ AD } & \multicolumn{5}{|c|}{ Espèces } & \multirow{2}{*}{\multicolumn{2}{|c|}{ Moyenne }} \\
\hline & & GB & PC & TB & $\mathbf{T E}$ & $\mathbf{T G}$ & & \\
\hline $\mathrm{C1}$ & 539,44 & - & - & - & - & 650,56 & 298,70 & $496,23(146,86)$ \\
\hline $\mathrm{C} 2$ & 492,22 & - & - & - & 288,52 & 677,41 & 473,70 & $482,96(137,65)$ \\
\hline $\mathrm{C3}$ & 630,37 & - & - & 130,37 & 241,48 & 537,78 & 426,67 & $393,33(184,59)$ \\
\hline $\mathrm{C4}$ & 483,70 & - & 168,89 & - & 317,04 & 594,81 & 539,26 & $420,74(212,14)$ \\
\hline C5 & 587,96 & - & 254,63 & - & - & 680,56 & 384,26 & $476,85(167,18)$ \\
\hline C6 & 598,52 & - & 172,59 & 376,30 & - & 709,63 & 487,41 & $468,89(185,19)$ \\
\hline C7 & 686,67 & 94,07 & - & 242,22 & 408,89 & 631,85 & 501,48 & $427,53(208,27)$ \\
\hline $\mathrm{C8}$ & 702,22 & 109,63 & 165,19 & 331,85 & 461,48 & 813,33 & 517,04 & $442,96(242,36)$ \\
\hline C9 & 604,81 & 160,37 & 401,11 & 271,48 & - & 734,44 & 530,74 & $450,49(195,59)$ \\
\hline C10 & 639,44 & 139,44 & 325,19 & - & - & 602,41 & 528,33 & $446,96(210,49)$ \\
\hline C11 & 543,70 & - & 339,63 & - & 413,70 & 617,41 & 395,19 & $461,93(102,57)$ \\
\hline $\mathrm{C12}$ & 474,07 & 157,41 & 351,85 & 388,89 & - & 527,78 & 444,44 & $390,74(118,72)$ \\
\hline $\mathrm{C13}$ & 416,30 & - & - & 268,89 & 379,26 & 471,85 & 360,74 & $379,41(67,04)$ \\
\hline C14 & 449,07 & - & - & 337,96 & - & 430,56 & 356,48 & $393,52(47,21)$ \\
\hline C15 & 401,11 & - & 225,19 & - & 318,52 & 465,93 & 354,81 & $353,11(80,74)$ \\
\hline Моу. & $\begin{array}{l}549,97 \\
(92,01)\end{array}$ & $\begin{array}{c}132,18 \\
(20,82)\end{array}$ & $\begin{array}{l}267,14 \\
(70,17)\end{array}$ & $\begin{array}{c}293,50 \\
(78,85)\end{array}$ & $\begin{array}{l}349,95 \\
(69,23)\end{array}$ & $\begin{array}{c}609,75 \\
(104,170)\end{array}$ & $\begin{array}{l}439,95 \\
(75,18)\end{array}$ & $\begin{array}{l}432,38 \\
(41,22)\end{array}$ \\
\hline
\end{tabular}

Légende: Loranthaceae: AD: Agelanthus dodoneifolius, GB: Globimetula braunii, PC: Phragmanthera capitata, TB: Tapinanthus bangwensis, TG: Tapinanthus globiferus, TE: Tapinanthus belvisii et TO: Tapinanthus ophiodes. Moy: Moyenne. 


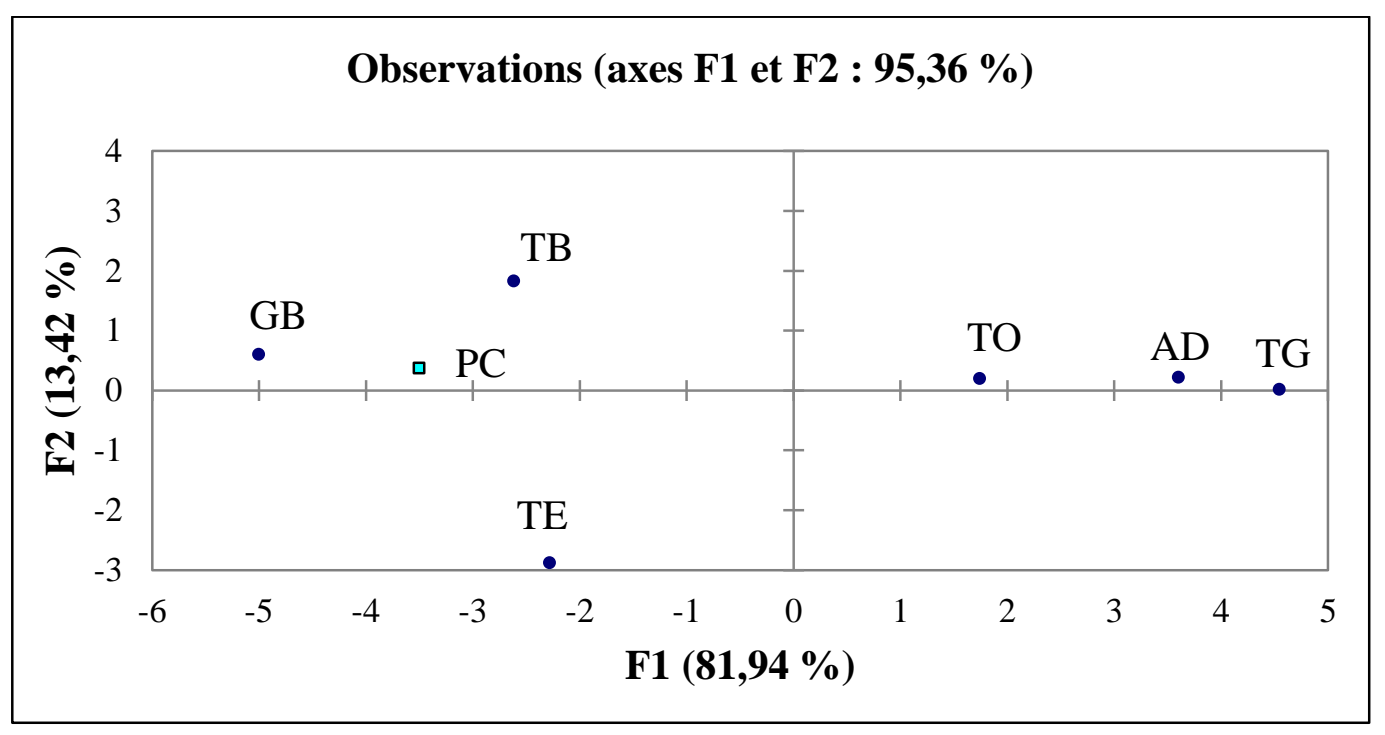

Figure 4: Dispersion des espèces de Loranthaceae sur l'ensemble des collines.

Légende: Loranthaceae: AD: Agelanthus dodoneifolius, GB: Globimetula braunii, PC: Phragmanthera capitata, TB: Tapinanthus bangwensis, TG: Tapinanthus globiferus, TE: Tapinanthus belvisii et TO: Tapinanthus ophiodes.

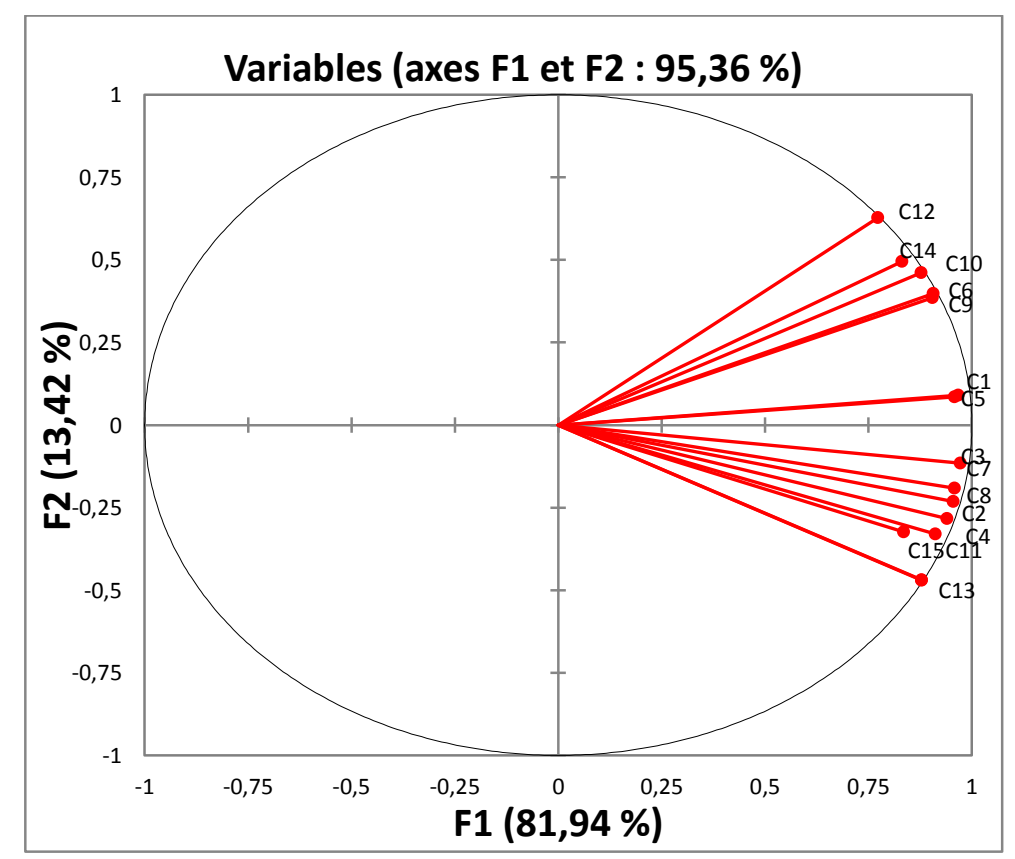

Figure 5: Corrélations entre les collines. 
Tableau 2: Indices de diversité et d'Equitabilité pour chacune des 15 collines.

\begin{tabular}{ccc}
\hline Collines & Indice de Shannon (bits) & Equitabilité de Pielou \\
\hline Colline 1 & 1,25 & 0,632 \\
Colline 2 & 1,32 & 0,640 \\
Colline 3 & 1,49 & 0,643 \\
Colline 4 & 1,53 & 0,642 \\
Colline 5 & 1,40 & 0,651 \\
Colline 6 & 1,52 & 0,651 \\
Colline 7 & 1,77 & 0,658 \\
Colline 8 & 1,90 & 0,763 \\
Colline 9 & 1,75 & 0,655 \\
Colline 10 & 1,74 & 0,649 \\
Colline 11 & 1,59 & 0,653 \\
Colline 12 & 1,70 & 0,648 \\
Colline 13 & 1,49 & 0,646 \\
Colline 14 & 1,38 & 0,645 \\
Colline 15 & 1,35 & 0,642 \\
\hline
\end{tabular}

Tableau 3: Catégories d'usage des Loranthaceae selon les réponses (\%) des enquêtés.

\begin{tabular}{cccccc}
\hline \multirow{2}{*}{ Phorophytes } & \multicolumn{5}{c}{ Catégories d'usage } \\
\cline { 2 - 6 } AA & TM & TD & I & MC & MR \\
\cline { 2 - 6 } CP & 32,49 & 54,85 & 8,44 & 45,57 & 8,86 \\
DM & 23,63 & 9,70 & 12,66 & 21,10 & 78,06 \\
KA & 13,08 & 10,13 & 13,50 & 22,78 & 19,41 \\
MI & 27,00 & 6,33 & 54,01 & 21,10 & 50,63 \\
SS & 47,26 & 38,82 & 61,60 & 43,04 & 82,70 \\
T & 37,97 & 27,43 & 64,56 & 28,69 & 80,17 \\
TI & 52,32 & 34,60 & 29,54 & 21,10 & 16,88 \\
V & 19,41 & 31,65 & 8,86 & 35,44 & 16,03 \\
XA & 46,41 & 8,86 & 54,85 & 14,77 & 65,40 \\
Moyenne & 24,05 & 36,29 & 18,99 & 14,35 & 37,55 \\
& 32,36 & 25,86 & 32,70 & 26,79 & 45,56
\end{tabular}

Légende: TM: Troubles mentaux, TD: Troubles digestifs, I: Infertilités, MC: Maladies chroniques, MR: Magico-religieux. Phorophytes portant les Loranthaceae: AA: Acacia albida, CP: Calotropis procera, DM: Diospyros mespiliformis, KA: Kigelia africana, MI: Mangifera indica, SS: Senna singueana, T: Terminalia sp., TI: Tamarindus indica, V: Vitex sp. et XA: Ximenia americana. 
Tableau 4: Valeurs d'usages des Loranthaceae.

\begin{tabular}{cccccc}
\hline Phorophytes & H & F & T & TP & VUT \\
\hline AA & 1,67 & 8,9 & 3,54 & 10,85 & 24,96 \\
CP & 2,25 & 12,51 & 2,87 & 11,62 & 29,25 \\
DM & 1,62 & 11,42 & 2,75 & 12,45 & 28,24 \\
KA & 2,19 & 9,65 & 2,76 & 7,72 & 22,32 \\
MI & 3,72 & 14,28 & 3,94 & 13,8 & 35,74 \\
SS & 3,94 & 11,63 & 4,21 & 14,63 & 34,41 \\
T & 2,2 & 12,39 & 2,58 & 7,87 & 25,04 \\
TI & 2,33 & 9,65 & 4,68 & 8,75 & 25,41 \\
V & 2,65 & 9,76 & 4,23 & 9,23 & 25,87 \\
XA & 2,05 & 11,43 & 2,89 & 9,75 & 26,12 \\
Moy/Ecart & $2,46 \pm 0,78$ & $11,16 \pm 1,67$ & $3,45 \pm 0,77$ & $10,67 \pm 2,42$ & $27,74 \pm 4,31$ \\
\hline
\end{tabular}

Légende: H: Homme, F: Femme, T: Tradithérapeute, TP: Tradipraticien. Phoropohytes des Loranthaceae: AA: Acacia albida, CP: Calotropis procera, DM: Diospyros mespiliformis, KA: Kigelia africana, MI: Mangifera indica, SS: Senna singueana, T: Terminalia sp., TI: Tamarindus indica, V: Vitex sp. et XA: Ximenia americana. Moy/Ecart: Moy: Moyenne, Ecart: Ecart-type.

Tableau 5: Revenu journalier, hebdomadaire, mensuel et annuel des ventes de Loranthaceae (brindilles et poudres) et des traitements des maladies avec les Loranthaceae.

\begin{tabular}{lcccc}
\hline \multirow{2}{*}{ Produits/ traitements } & \multicolumn{4}{c}{ Revenu en FCFA } \\
\cline { 2 - 5 } & Journalier & Hebdomadaire & Mensuel & Annuel \\
\hline Brindilles & 200 & 1000 & 70000 & 250000 \\
Poudres & 300 & 1500 & 50000 & 200000 \\
Maladies traitées & 500 & 3000 & 90000 & 350000 \\
Cas mystiques & 1000 & 5000 & 100000 & 400000 \\
Total & 2000 & 10500 & 310000 & 1200000 \\
\hline
\end{tabular}

\section{DISCUSSION}

\section{Diversité des Loranthaceae des Monts Mandara}

Les Loranthaceae des Monts Mandara de la Région de l'Extrême-Nord du Cameroun renferment 4 genres (Agelanthus, Globimetula, Phragmanthera et Tapinanthus) et 7 espèces de Loranthaceae parasites des plantes ligneuses qui sont: A. dodoneifolius, G. braunii, $P$. capitata, T. bangwensis, T. globiferus, $T$. belvisii et $T$. ophiodes. Ce nombre d'espèces inventoriées est inférieur aux 9 espèces inventoriées par Souare et al. (2020) dans la plaine du Diamaré de la Région de l'ExtrêmeNord du Cameroun. Mais ce nombre est supérieur aux 5 espèces récoltées sur les plantes ligneuses par Mapongmetsem et al. (1998) dans les Savanes soudano-guinéennes de l'Adamaoua, au Cameroun. Les différences observées entre le nombre d'espèces seraient dues aux milieux écologiques étudiés et aux conditions climatiques des sites prospectés. En effet, notre étude est réalisée sur les montagnes avec des conditions climatiques particulières alors que celles de ces auteurs ont été conduites dans la plaine et en basses altitudes.

Les exigences écologiques des 7 espèces de Loranthaceae inventoriées sur les Monts Mandara sont variables en termes de distribution sur les collines étudiées. Parmi ces espèces, certaines se rencontrent sur toutes les collines, à savoir A. dodoneifolius, $T$. 
globiferus et $T$. ophiodes tandis que d'autres comme T. belvisii, T. bangwensis, P. capitata et $G$. braunii sont confinées sur des collines bien précises des Monts Mandara. La distribution géographique de ces trois premières espèces n'est liée à aucune exigence écologique particulière en zone montagneuse ; c'est ce qui explique leur présence sur toutes les collines. Par contre, T. belvisii, T. bangwensis, $P$. capitata et $G$. braunii semblent présenter des exigences du point de vue écologique. Ces résultats confirment les travaux de Amon et al. (2010) qui ont rapporté que certaines espèces des Loranthaceae comme $T$. bangwensis et $P$. capitata se rencontrent partout en Côte d'Ivoire, surtout sur terre ferme, alors que T. belvisii est confinée, au Sud du pays sur le littoral. Pour Jiofack et al. (2009) et Amon et al. (2010), l'expansion importante de certaines espèces de Loranthaceae d'une localité à l'autre serait en fonction de la variabilité des conditions écologiques des milieux.

Les collines influencent la répartition des Loranthaceae aussi bien par le nombre d'espèces trouvées que par leur abondance sur les Monts Mandara. En effet, la colline 8 riche de 7 espèces de Loranthaceae inventoriées tandis que la colline 1 avec trois espèces parasites, est la moins riche. Aussi est-il indiqué une abondance en nombre d'individus de Loranthaceae recensés avec des densités respectives de 496,23 et 482,96 ind./ha sur les collines 1 et 2 , tandis que la colline 15 enregistre une faible abondance en nombre d'individus des parasites avec une densité de 353,11 ind./ha. Ces différences obtenues entre les collines pourraient s'expliquer par les conditions écologiques du milieu et par les activités anthropiques. En effet, la colline 8 est située au centre de la chaîne montagneuse avec des conditions environnementales favorables au développement des espèces de Loranthaceae. C'est pourquoi sur la colline 8, le nombre d'espèces est élevé. Par contre les collines 1 et 15 , localisées aux extrémités de la chaîne montagneuse sont exposées aux vents et aux rayonnements solaires plus intenses et les conditions écologiques de ces collines ne sont donc favorables à l'expansion des espèces de Loranthaceae. La faible densité obtenue sur la colline 15 par rapport à la colline 1 s'explique par les activités anthropiques plus élevées sur la colline 15 que la colline 1 .

\section{Importance socio-économique des} Loranthaceae des Monts Mandara

Les populations des Monts Mandara connaissent bien les Loranthaceae puisqu'elles les désignent par différentes appellations à partir de leurs dialectes locaux. Cette connaissance des Loranthaceae par les différentes ethnies a été déjà confirmée par Azo'o et al. (2013) en étudiant le parasitisme et l'ethnobotanique des Loranthaceae à Lokomo à l'Est du Cameroun. La présente étude a révélé l'importance socio-économique des Loranthaceae dans l'existence des tribus constitutives des Arrondissements des Monts Mandara à travers des usages en pharmacopée et la commercialisation des espèces inventoriées. Ces résultats confirment les résultats de Aka et al. (2016) et de Soumaila et al. (2017) qui ont déjà signalé que les Loranthaceae parasites des plantes ligneuses sont diversement utilisées par de nombreuses populations locales dans leurs soins de santé. Les propriétés des espèces de Loranthaceae sont utilisées par les paysans des Monts Mandara pour traiter par des pratiques magicoreligieuses des maux tels que les infertilités, les troubles mentaux, les troubles digestifs et autres affections chroniques. Ces résultats corroborent ceux de Azo'o et al. (2013) qui ont déjà démontré que les espèces de Loranthaceae sont utilisées pour traiter les attaques nerveuses, la rougeole, l'épilepsie, les plaies chroniques, les abcès et opérer des pratiques mystiques. Arbonnier (2009) a montré en Afrique de l'Ouest que les plantes épiphytes et parasites comme les Loranthaceae sont traditionnellement reconnues comme les plantes ayant des pouvoirs magico-religieux très importants et contiennent des propriétés pharmacodynamiques de la plante hôte. Elles sont notamment utilisées pour soigner les maladies mentales. Ahamide et al. (2017) rapportent aussi que les espèces de 
Loranthaceae, parasites des plantes ligneuses sont très utilisées dans le traitement de plusieurs maux parmi lesquels la stérilité et les fausses couches.

Les résultats des enquêtes ont montré que les connaissances traditionnelles des Loranthaceae dans les Monts Mandara varient avec le genre et le groupe des exploitants. Ces résultats confirment les travaux de Gouwaknnou et al. (2011). Les espèces hôtes des Loranthaceae telles que Mangifera indica, Senna singueana, Calotropis procera et Diospyros mespiliformis ont enregistré des valeurs d'utilisation traditionnelle les plus élevées. Ces résultats sont différents de ceux obtenus par Lougbegnon et al. (2011), qui ont identifié Psidium guajava, Dialium guineense, Senna alata, Uvaria chamae et Cola millenii comme les espèces ayant les valeurs d'usages les plus élevées. La différence observée serait due à la composition floristique et des facteurs climatiques des zones d'étude. En effet, ces auteurs ont mené leur étude dans la réserve forestière marécageuse alors que la présente étude s'est déroulée sur les collines avec des conditions climatiques différentes.

Les Loranthaceae constituent une source importante de revenu économique pour les utilisateurs. Les espèces de Loranthaceae sont vendues sur les marchés des zones urbaines et rurales par les tradithérapeutes et les tradipraticiens. Elles génèrent pour les utilisateurs des revenus mensuels variables. Ces résultats confirment les travaux de Chupezi et al. (2009) en Afrique du Sud.

\section{Conclusion}

Les Loranthaceae des collines des Monts Mandara comprennent 4 genres (Agelanthus, Globimetula, Phragmanthera et Tapinanthus) et 7 espèces parasites, à savoir: A. dodoneifolius, G. braunii, P. capitata, T. bangwensis, T. belvisii, T. globiferus et $T$. ophiodes. La colline 8 dans l'Arrondissement de Mokolo présente la plus grande richesse spécifique avec toutes les 7 espèces parasites et la colline 1 située dans l'Arrondissement de Mora enregistre le plus faible nombre avec 3 espèces (A. dodoneifolius, T. globiferus et $T$. ophiodes). L'analyse des caractéristiques médicinales et socio-économiques des Loranthaceae montre que celles-ci sont utilisées dans les pratiques magico-religieuses $(45,56 \%)$ pour traiter les infertilités masculines et féminines $(32,70 \%)$, les troubles mentaux $(32,36 \%)$, les affections chroniques $(26,79 \%)$ et les troubles digestifs $(25,86 \%)$. La commercialisation des Loranthaceae génère des revenus mensuels variables aux populations des Monts Mandara. L'exploitation durable des Loranthaceae reste d'un grand intérêt pour ses utilisateurs dans la Région de l'Extrême-Nord du Cameroun.

\section{CONFLIT D'INTERETS}

Les auteurs déclarent qu'ils n'ont aucun conflit d'intérêts.

\section{CONTRIBUTIONS DES AUTEURS}

DM a conduit l'étude et développé le manuscrit. SK et IA ont lu et fait de commentaires afin d'améliorer la qualité scientifique du manuscrit.

\section{REMERCIEMENTS}

Nous remercions sincèrement toutes les populations, les autorités traditionnelles et administratives des Monts Mandara pour leur active contribution à la collecte des données pour cette étude.

\section{REFERENCES}

Adomou AC, Yedomonhan H, Djossa B, Legba SI, Oumorou M, Akoegninou A. 2012. Etude ethnobotanique des plantes médicinales vendues dans le marché d'Abomey-Calavi au Bénin. International Journal of Biological and Chemical Sciences, 6(2): 745-772. DOI: $10.4314 / \mathrm{ijbcs.v6i2.18}$

Ahamide IDY, Monique GT, Adomou AC, Houenon JG, HounnankponY, Akpovi AA. 2015. Diversité, impacts et usages des Loranthaceae parasites de Cola nitida (Vent.) Schott. \& Endl. au Sud-Bénin. International Journal of Biological and Chemical Sciences, 9(6): 2859-2870. DOI: $10.4314 / \mathrm{ijbcs.v9i6.26}$ 
Ahamide IDY, Monique GT, Hounnankpon Y, Aristide CA. 2017. Diversité des Loranthaceae et leur impact sur Vitellaria Paradoxa C.F.Gaertn. : un fruitier à grande valeur socio-économique au Nord-Bénin. European Scientific Journal, 24(13): 1857-7431. DOI: https://doi.org/10.19044/esj.2017.v 13n24p217.

Aka R, Danho FRN, Klotioloma C, Kouamé F, N'guessan, Kebe IB. 2016. Inventaire et distribution des espèces de végétaux parasites et épiphytes du cacaoyer en Côte d'Ivoire. Journal of Animal \& Plant Sciences, 2: 5010-5020.

Akoègninou A, van der Burg WJ, van der Maesen LJG. 2006. Flore Analytique du Bénin. Brackhuys Publishers: Wageningen.

Amon ADE, Soro D, N'guessan K, Traoré D. 2010. Les Loranthaceae: plantes vasculaires des arbres et arbustes, au SudEst de la Côte d'Ivoire. Journal of Applied Biosciences, 25: 1565-1572.

Amon ADE, Soro D, Traoré D. 2015. Evaluation de l'infestation des Loranthacées sur les ligneux des agroécosystèmes de la région du SudComoé (Côte d'Ivoire). International Journal of Biological and Chemical Sciences, $\quad$ 9(4): $\quad 1822-1834$. DOI: 10.4314/ijbcs.v9i4.8

Amon ADE, Seguena F, Soro K, Soro D, Koffi N. 2017. Ethnobotany study of Loranthaceae, hemiparasitic plants used in traditional medicine by population, in the Sud-Comoe region (Côte d'Ivoire). Journal of Medicinal Plants Studies, 5(5): 217-224.

Arbonnier M. 2009. Arbres, arbustes et lianes des zones sèches Afrique de l'Ouest. Editions Quae-MNHN : Versailles.

Azo'o J, Tchatat M, Mony R, Dibong SD. 2013. Parasitisme et ethnobotanique des Loranthaceae à Lokomo (EstCameroun). Journal of Animal and Plant Sciences, 2(19): 29223-2932.

Boussim IJ. 2002. Les phanérogames parasites du Burkina Faso : Inventaire, taxonomie, écologie et quelques aspects de leur biologie. Cas particulier des Loranthaceae parasites du karité. Thèse de Doctorat d'Etat es Sciences Naturelles, Université d'Ouagadougou, Burkina Faso, $306 \mathrm{p}$.

BUCREP. 2005. Rapport national sur l'état de la population, enjeux et défis d'une population de 20 millions au Cameroun. BUCREP, Cameroun, $106 \mathrm{p}$.

Chupezi TJ, Ndoye O, Tchatat M, Chikamai B. 2009. Processing and Marketing of Nonwood Forest Products: Potential Impacts and Challenges in Africa. Discov. Innov., 21(1): 60-65. DOI: $10.4314 /$ dai.v21i3.48214

Dibong SD, Engone ONL, Priso RJ, Taffouo VD, Fankem H, Sallé G, Amougou A. 2009. Les Loranthaceae: un atout pour l'essor de la pharmacopée traditionnelle au Cameroun. International Journal of Biological and Chemical Sciences, 3(4): 746-754. DOI: 10.4314/ijbcs.v3i4.47185

Dibong SD, Biyon BN, Engone N. 2010. Fautil éradiquer les Loranthaceae sur les ligneux à fruits commercialisés de la région littorale du Cameroun ? International Journal of Biological and Chemical Sciences, 4(3): 555-562. DOI: $10.4314 /$ ijbcs.v4i3.60450

Gerhard MK. 2003. The way of the beer: ritual reenactment of historyamong the Mafa, terracefarmers of the Mandara Mountains (North Cameroun). Mandaras Publishing: Londres.

Gouwakinnou GN, Lykke AM, Assogbadjo AE, Sinsin B. 2011. Local knowledge, pattern and diversity of use of Sclerocarya birrea. Journal of Ethnobiology and Ethnomedicine, 7(8): 1746-4269. DOI: 10.1186/1746-4269-7-8 Houénon GJ. 2012. Les Loranthaceae des zones guinéennes et soudano-guinéenne au Bénin et leur impact sur les plantations agrumicoles. Thèse de Doctorat, Université d'Abomey-Calavi, Bénin, 133 p.

Jiofack T, Ayissi I, Fokunang C, Guedje N, Kemeuze V. 2009. Ethnobotany and 
phytomedicine of the upper Nyong Valley forest in Cameroon. African Journal of Pharmacy and Pharmacology, 3(4): 144-150.

Koffi AA, Kouassi FA, N'goran SBK, Soro D. 2014. Les Loranthaceae, parasites des arbres et arbustes : cas du département de Katiola, au nord de la Côte d'Ivoire. International Journal of Biological and Chemical Sciences, 8(6): 2552-2559. DOI: $10.4314 /$ ijbcs.v8i6.17

Kouyaté AM. 2005. Aspects ethnobotaniques et étude de la variabilité morphologique, biochimique et phénologique de Detarium microcarpum guill. \& perr. au Mali. Thèse en Engineering, Faculty of Bioscience Engineering, Ghent University, Belgium, $188 \mathrm{p}$.

Lougbegnon TO, Tente BAH, Amontcha M, Codjia JTC. 2011. Importance culturelle et valeur d'usage des ressources végétales de la réserve forestière marécageuse de la vallée de Sitatunga et zones connexes. Bulletin de la Recherche Agronomique du Bénin, 4: 35-46.

Maïnam F. 1999. Modelling soil erodibility in semi arid zone of Cameroon, Assessment of interill erodibility parameter for mapping soil erosion hazard by means of GIS techniques in the Gawar area. Ph.D. Thesis, Faculty of Sciences, University of Ghent, Netherlands, 387 p.
Mapongmetsem PM, Motalindja M, Nyomo H. 1998. Eyes on the enemy. Identifying parasitic plants of wild fruit trees in Cameroon. Agroforest Today, 10(3): 1011.

Mony R, Tchatat M, Massako F, Dibong SD. 2014. Parasitisme du safoutier par les Tapinanthus au plateau de Logbessou (Douala, Cameroun). Tropicultura, 32(4): 177-182.

Nabila T, Abdelkrim EL, Basti, Lahcen Z, Atmane R, Allal D. 2012. Etude ethnobotanique des plantes médicinales dans la Province de Settat (Maroc). Journal of Forestry Faculty, 12(2): 192208.

Polhill R, Wiens D. 1998. Mistletoes of Africa. The Royal Botanic Gardens: Kew.

Souare K, Baye-Niwah C, Hamawa Y, Tezore BP. 2020. Local Uses and Parasitism of Loranthaceae in Sudano-Sahelian Zone of Cameroon: Case of Diamare Plain in Far North Region. International Journal of Sciences, $\quad 9$ : $\quad 1-8 . \quad$ DOI: 10.18483/ijSci.2350

Soumaila M, Barmo S, Boube M, Saley K, Hassane BI, Ali M, Kalid I, Mahamane S. 2017. Inventaire et gestion des plantes médicinales dans quatre localités du Niger. European Scientific Journal, 24(13): $1857-7431$ DOI: 10.19044/esj.2017.v13n24p498 